\title{
SHIFT AND BROADENING OF THE VIBRATIONAL MODE FOR A DIATOMIC MOLECULE ADSORBED ON A METAL SURFACE
}

\author{
G.W. FORD \\ Department of Physics, The University of Michigan, Ann Arbor, Michigan 48109, USA
}

and

\section{W.H. WEBER}

Research and Engineering Staff, Ford Motor Company, Dearborn, Michigan 48121, USA

Received 9 November 1982; accepted for publication 7 February 1983

\begin{abstract}
The shift and broadening of the vibrational frequency of a diatomic molecule adsorbed on a metal surface are calculated on the basis of electromagnetic interactions. Finite molecular size and nonlocal metal response are included in a calculation of the response function, whose pole is the complex normal mode frequency. Finite molecular size is described by a model of a point-like vibrational mode within a polarizable sphere. Nonlocal metal response is described by a semiclassical infinite barrier model with a Lindhard dielectric constant generalized to include core polarization and finite electron lifetime. When applied to the case of $\mathrm{CO}$ adsorbed on $\mathrm{Cu}$ and if the molecule is positioned so as to fit the observed width, it is found the predicted shift is a factor of three or more greater than that observed.
\end{abstract}

\section{Introduction}

The problem of the electromagnetic interaction of an adsorbed molecule with a metal surface is one of great interest. Here we treat one aspect of this problem, the question of the shift and broadening of molecular vibrational modes. Previous discussions of the shift in the vibrational frequency of an adsorbed molecule due to the image forces in the metal have either treated the metal as a perfect reflector [1,2] or have included static screening effects via a Fermi-Thomas approximation [3,4], or via a density functional calculation. In these calculations the molecule has been treated either as a polarizable point [1-3], or as a pair of separated charges [4,5]. In this paper we include two important aspects of the interactions which are missing in these treatments; the finite size of the polarizable electron cloud of the molecule and the nonlocal dynamical response of the metal. An important feature of the nonlocal dynamical response is the absorption by electron-hole pairs. This gives rise to 
an associated damping of the vibrational mode. This absorption has been calculated for a pointlike dipole with given frequency above a nonlocal metal [6-9]. But such a calculation, besides neglecting the effects of finite molecular size, also neglects the response of the dipole to the reflected fields. It is exactly this response which is responsible for the electromagnetic shift in vibrational frequency mentioned above. It is therefore desirable to calculate the shift and broadening within a single coherent framework. This is one of the aims of this paper.

In this work, we calculate the shift and broadening for a diatomic molecule above a metal surface and oriented perpendicular to the surface. We describe the molecule by a generalization of the finite sphere model described in ref. [9], thus taking into account finite size. The nonlocal metal response is described by a semiclassical infinite barrier (SCIB) model with a Lindhard dielectric constant. We then calculate the response function of the system and identify the complex normal mode frequencies with its poles. The shift and width of the vibrational frequency are associated with the real and imaginary part of the complex normal mode frequency, respectively.

In section 2 we describe our model of a finite-size diatomic molecule as a vibrational mode within a polarizable sphere. In section 3 we then calculate the response function for the system corresponding to this model molecule placed above a nonlocal metal half-space. We obtain an explicit formula for the complex frequency corresponding to the vibrational mode. We present in this same section numerical results for the case of $\mathrm{CO}$ adsorbed on $\mathrm{Cu}$. An important conclusion is that the core polarization term in the dielectric constant has a large effect, because it is unscreened at large wave vectors. This term has heen neglected in previous treatments of this problem. Before comparing the predicted shift and broadening with experiment, in section 4 we discuss other expected contributions to the shift, the mechanical shift and the chemical shift due to changes in the bond strength. Finally in section 5 we discuss our results in comparison with experiment and with other calculations.

\section{Polarizable sphere model of a diatomic molecule}

Our model is that of a sphere of radius $a$ and dielectric constant $\epsilon_{3}$ with an oscillating point dipole $\mu$ at its center. The idea is that the sphere represents the polarizable electron cloud and the point dipole represents the charge separation induced by the nuclear motion. The equation of motion of the dipole is assumed to be

$M \ddot{\mu}+M \omega_{0}^{2} \mu=q^{2} E(t)$,

where $E$ is the field at the dipole. The parameters $M, q$, and $\omega_{0}$ are, respectively, the reduced mass, effective charge, and (bare) frequency of the 
oscillator. The response of this model molecule to an arbitrary applied field is described by the multipole polarizabilities, which we now construct.

If a 2 '-pole electric field with potential of the form

$\Phi_{\text {applied }}=-A_{l} r^{l} P_{l}(\cos \theta) e^{-i \omega r}$

acts on the molecule, the resulting fields will have potential of the form

$\Phi= \begin{cases}A_{l}\left(-r^{\prime}+\frac{\alpha_{l}}{\epsilon_{1} r^{l+1}}\right) P_{l}(\cos \theta) \mathrm{e}^{-\mathrm{i} \omega t}, & r>a, \\ \left(C_{l} r^{\prime}+\frac{\mu}{\epsilon_{3} r^{2}} \delta_{l, 1}\right) P_{l}(\cos \theta) \mathrm{e}^{-\mathrm{i} \omega t}, & r<a,\end{cases}$

where the orientation of the dipole at the center of the sphere has been chosen as the polar axis. Here $\alpha_{i}$ is the $2^{\prime}$-pole polarizability and $\epsilon_{1}$ is the dielectric constant of the medium surrounding the sphere. For $l>1$, the requirements of continuity of $\Phi$ and $\epsilon \partial \Phi / \partial r$ at $r=a$ give the same results for the multipole polarizability as for the sphere without the dipole [9],

$\alpha_{l}=\frac{\epsilon_{1} l\left(\epsilon_{3}-\epsilon_{1}\right)}{l \epsilon_{3}+(l+1) \epsilon_{1}} a^{2 l+1}, \quad l>1$.

For $l=1$ we find from continuity of $\phi$

$A_{1}\left(-a+\frac{\alpha_{1}}{\epsilon_{1} a^{2}}\right)=C_{1} a+\frac{\mu}{\epsilon_{3} a^{2}}$,

while from continuity of $\epsilon \partial \Phi / \partial r$,

$-A_{1}\left(E_{1}+\frac{2 \alpha_{1}}{a^{3}}\right)=C_{1} \epsilon_{3}-\frac{2 \mu}{a^{3}}$.

In addition we have from the equations of motion (1) the relation

$M\left(\omega_{0}^{2}-\omega^{2}\right) \mu=-q^{2} C_{1}$.

Eqs. (5), (6), and (7) are three inhomogeneous linear equations for $\alpha_{1}, \mu$, and $C_{1}$. In our further discussion we need only $\alpha_{1}$, which is given by

$\alpha_{1}=\epsilon_{1} \frac{\epsilon_{3}-\epsilon_{1}+\frac{2 \epsilon_{3}+\epsilon_{1}}{\epsilon_{3} a^{3}} \frac{q^{2}}{M\left(\omega_{0}^{2}-\omega^{2}\right)}}{\epsilon_{3}+2 \epsilon_{1}+2 \frac{\epsilon_{3}-\epsilon_{1}}{\epsilon_{3} a^{3}} \frac{q^{2}}{M\left(\omega_{0}^{2}-\omega^{2}\right)}} a^{3}$.

The observed vibrational frequency $\omega_{v}$ corresponds to the vanishing denominator of this expression,

$\omega_{v}^{2}=\omega_{0}^{2}+2 \frac{\epsilon_{3}-\epsilon_{1}}{\epsilon_{3}\left(\epsilon_{3}+2 \varepsilon_{1}\right)} \frac{q^{2}}{M a^{3}}$. 
This gives the renormalization of the bare vibrational frequency $\omega_{0}$ arising from the electronic cloud. Eliminating the unobservable frequency $\omega_{0}$ between (8) and (9) we can write the dipole polarizability in the form

$\alpha_{1}(\omega)=\alpha_{\mathrm{e}}+\frac{\alpha_{v}}{1-\left(\omega / \omega_{\mathrm{v}}\right)^{2}}$,

where

$\alpha_{\mathrm{e}}=\epsilon_{1} \frac{\epsilon_{3}-\epsilon_{1}}{\epsilon_{3}+2 \epsilon_{1}} a^{3}$

is the electronic polarizability and

$\alpha_{v}=\frac{9 \epsilon_{1}^{2}}{\left(\epsilon_{3}+2 \epsilon_{1}\right)^{2}} \frac{q^{2}}{M \omega_{v}^{2}}$

is the vibrational contribution to the polarizability.

The expression (10) is the familiar phenomenological form for the dipole polarizability of a molecule with a vibrational degree of freedom. As in the phenomenological treatments we determine the parameters $\alpha_{\mathrm{e}}$ and $\alpha_{\mathrm{v}}$ from the measured high and low frequency polarizability and take $\omega_{v}$ to be the vibrational frequency of the isolated molecule. In addition, we eliminate $\epsilon_{3}$ between (11) and (4) to express the higher multipole polarizabilities in terms of $\alpha_{\mathrm{e}}$. The result can be combined with (10) in the general form

$\alpha_{l}(\omega)=\epsilon_{1} \frac{3 l \alpha_{\mathrm{e}}}{(l-1) \alpha_{\mathrm{e}}+(2 l+1) \epsilon_{1} a^{3}} a^{2 l+1}+\frac{\alpha_{\mathrm{v}}}{1-\left(\omega / \omega_{\mathrm{v}}\right)^{2}} \delta_{l, 1}$.

We view this form, which was derived on the basis of our simple model, as a phenomenological form with parameters to be fitted to measured values. The radius $a$ is perhaps the least well-determined and will be given a value that seems reasonable. Other models of the electronic cloud could be introduced to determine the form of the electronic contribution to $\alpha_{l}$; for a discussion see ref. [9] where it is argued that the dielectric model gives a reasonable approximation to the form of $\alpha_{l}$ for real molecules.

Efrima and Metiu have introduced a different finite-size molecular model, in which the vibrational polarizability is described by a pair of point charges oscillating about a finite separation and the electronic polarizability is described by a polarizable point at the center of the molecule [4,5]. Their model and ours might be viewed as two extremes, we treat the vibrator as pointlike and ascribe the finite size to the finite size of the valence electron cloud responsible for the electronic polarizability, they on the other hand treat the electron cloud as pointlike and ascribe the finite size to the finite separation of the vibrating ion cores. We feel that for our purpose, which is to calculate the electromagnetic effects, our model is more appropriate, since the outer regions 
of the molecule are in fact occupied by the valence electrons. Moreover, our calculations for this and other problems have shown us that all finite size effects disappear very rapidly as the molecule is moved away from the surface [9]. The vibrator, being more distant than the electron cloud, should therefore be more pointlike.

\section{Response of a molecule placed above a nonlocal metal surface}

Consider now as in fig. 1 a molecule placed with its center a distance $d$ above a metal surface, and subject to an oscillating electric field $E_{0} \mathrm{e}^{-\mathrm{i} \omega t}$ directed perpendicular to the surface. The induced dipole moment at frequency $\omega, \mu(\omega) \mathrm{e}^{-\mathrm{i} \omega t}$, is given by (see eq, (28) of ref. [9])

$\mu(\omega)=\epsilon_{1} G(\omega) E_{0}$,

where $G(\omega)$ is the response function, given by

$G(\omega)=\operatorname{cof}\left(M_{11}\right) / \operatorname{det}(M)$.

Here the denominator is the determinant of an infinite matrix $M$ and the numerator is the cofactor of the 11 element of $\mathrm{M}$. The elements of the matrix $M$ are given by

$M_{l^{\prime}}=\frac{\epsilon_{1}}{\alpha_{l}(\omega)} \delta_{l^{\prime}}-\frac{F_{l^{\prime}}}{l ! l^{\prime} !}, \quad l, l^{\prime}=1,2, \ldots$,

where $\alpha_{l}$ is the multipole polarizability of the sphere and

$F_{l \prime}(\omega, d)=\int_{0}^{\infty} \mathrm{d} p r_{12}^{\mathrm{p}}(p, \omega) p^{i+l^{\prime}} \mathrm{e}^{-2 p d}$,

in which $r_{12}^{\mathrm{p}}(p, \omega)$ is the quasistatic retlection coerricient ror p-polarized electromagnetic waves of frequency $\omega$ incident on the surface, with $p$ the component of the wave vector parallel to the surface. We see therefore that if we have a molecular model for which we can calculate $\alpha_{l}(\omega)$ and a model for

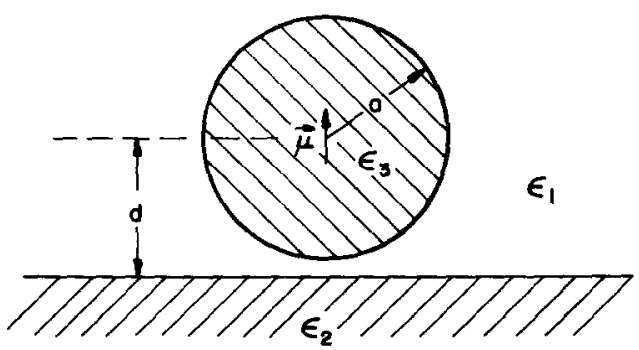

Fig. 1. Schematic drawing of adsorbed molecule above a metal half-space. 
the metal for which we can calculate the reflection coefficient $r_{12}^{\mathrm{p}}$, then we have a prescription for calculating $G(\omega)$. In this paper we use the molecular model described in section 2 and for the metal the SCIB model for which the calculation of $r_{12}^{\mathrm{p}}$ is described in detail in ref. [9].

The complex resonance frequencies of the adsorbed molecule correspond to the poles of $G(\omega)$. That is,

$1 / G\left(\omega_{\mathrm{r}}\right)=0$.

This relation can be rearranged into a form in which the vibrational parameters are made explicit by noting first that we can write (13) in the form

$\frac{1}{\alpha_{l}(\omega)}=\frac{(l-1) \alpha_{\mathrm{e}}+(2 l+1) \epsilon_{1} a^{3}}{3 l \epsilon_{1} \alpha_{\mathrm{e}} a^{2 l+1}} \frac{\alpha_{\mathrm{v}}}{\alpha_{\mathrm{e}}\left(\alpha_{\mathrm{e}}+\alpha_{\mathrm{v}}\right) \omega_{\mathrm{v}}^{2}-\alpha_{\mathrm{e}} \omega^{2}} \delta_{l, 1}$.

Using this in the expression (16) for the matrix elements and imagining the expansion of $\operatorname{det}(M)$ according to the elements of the first row, we readily see that

$\frac{1}{G(\omega)}=\frac{1}{G_{\mathrm{e}}(\omega)}-\frac{\epsilon_{1} \alpha_{\mathrm{v}}}{\alpha_{\mathrm{e}}} \frac{\omega_{\mathrm{v}}^{2}}{\left(\alpha_{\mathrm{e}}+\alpha_{\mathrm{v}}\right) \omega_{\mathrm{v}}^{2}-\alpha_{\mathrm{e}} \omega^{2}}$,

were $G_{\mathrm{e}}(\omega)$ is the purely electronic response function, given by (15) with $\alpha$, replaced with the electronic contribution alone, obtained by setting $\alpha_{v}=0$ in (13). The point of this separation is that $G_{\mathrm{e}}(\omega)$ is a slowly varying function of $\omega$, with no resonance structure at $\omega \approx \omega_{\mathrm{v}}$. Therefore, so long as the resonance frequency $\omega_{\mathrm{r}}$ is near $\omega_{\mathrm{v}}$, i.e., the shift is small, we can replace $G_{\mathrm{e}}(\omega) \rightarrow G_{\mathrm{e}}\left(\omega_{\mathrm{v}}\right)$ in (20). Using this in (18) we can solve explicitly for $\omega_{r}$ to get

$\omega_{r}^{2}=\omega_{v}^{2}\left[1+\frac{\alpha_{v}}{\alpha_{e}}\left(1-\frac{\epsilon_{1} G_{e}\left(\omega_{v}\right)}{\alpha_{e}}\right)\right]$.

The real part of $\omega_{r}$ is interpreted as the shifted vibrational frequency, the imaginary part as the width. So long as the shift is small we can write the relative shift as

$\frac{\Delta \omega}{\omega_{v}} \equiv \operatorname{Re}\left\{\frac{\omega_{\mathrm{r}}-\omega_{\mathrm{v}}}{\omega_{\mathrm{v}}}\right\} \approx \frac{\alpha_{\mathrm{v}}}{2 \alpha_{\mathrm{e}}} \operatorname{Re}\left\{1-\frac{\epsilon_{1} G_{\mathrm{e}}\left(\omega_{\mathrm{v}}\right)}{\alpha_{\mathrm{e}}}\right\}$,

and the relative half-width as

$$
\frac{\gamma}{\omega_{\mathrm{v}}} \equiv-\operatorname{Im}\left\{\frac{\omega_{\mathrm{r}}-\omega_{\mathrm{v}}}{\omega_{\mathrm{v}}}\right\} \approx \frac{\epsilon_{1} \alpha_{\mathrm{v}}}{2 \alpha_{\mathrm{e}}^{2}} \operatorname{Im}\left\{G_{\mathrm{e}}\left(\omega_{\mathrm{v}}\right)\right\}
$$

If the molecule were modeled as a polarizable point these same formulas would apply excepting that only the dipolar polarizability $\alpha_{1}$ would be nonzero $\left(\alpha_{l}=0, l>1\right)$. In this case

$G_{\mathrm{e}}(\omega)^{-1}=\frac{\epsilon_{1}}{\alpha_{l}}-F_{11}(\omega, d) \quad$ (point model). 
When this expression is used in (23) we get

$$
\begin{aligned}
& \frac{\Delta \omega}{\omega_{\mathrm{v}}} \approx-\frac{\alpha_{\mathrm{v}}}{2} \operatorname{Re}\left\{\frac{F_{11}(\omega, d)}{\epsilon_{1}-\alpha_{\mathrm{e}} F_{11}(\omega, d)}\right\} \quad \text { (point model). } \\
& \frac{\gamma}{\omega_{\mathrm{v}}} \approx \frac{\alpha_{\mathrm{v}}}{2} \operatorname{Im}\left\{\frac{F_{11}(\omega, d)}{\epsilon_{1}-\alpha_{\mathrm{e}} F_{11}(\omega, d)}\right\}
\end{aligned}
$$

In a recent publication Persson calculates the decay rate $r^{-1}=2 \gamma$ for a radiating point dipole above a metal $[6,9,10]$. His result is obtained from (25) if we first neglect $\alpha_{\mathrm{e}} F_{11}$ in the denominator (i.e., neglect molecular polarization by the induced fields from the metal) and then make the identification 2 $\omega_{v} \alpha_{v}=\mu^{2}$, where $\mu$ is twice the transition dipole moment between the ground and first excited states for a harmonic oscillator. We see therefore that Persson's formulation, aside from neglecting finite molecular size, does not take account of the renormalization of the dipole strength arising from the reflected fields. This renormalization is just what in another context is called the image-enhancement effect [9].

If, in addition to neglecting finite molecular size, as in (25), we treat the metal as a local dielectric medium with dielectric constant $\epsilon_{2}(\omega)$, then $r_{12}^{\mathrm{p}}=\left(\epsilon_{2}\right.$ $\left.-\epsilon_{1}\right) /\left(\epsilon_{2}+\epsilon_{1}\right)$ and $F_{11}=\mathrm{r}_{12}^{\mathrm{p}} / 4 d^{3}$. Eq. (25) then becomes

$$
\begin{aligned}
& \frac{\Delta \omega}{\omega_{v}}=-\frac{1}{2} \alpha_{\mathrm{v}} \operatorname{Re}\left\{\frac{\left(\epsilon_{2}-\epsilon_{1}\right) /\left(\epsilon_{2}+\epsilon_{1}\right)}{4 d^{3} \epsilon_{1}-\alpha_{\mathrm{e}}\left(\epsilon_{2}-\epsilon_{1}\right) /\left(\epsilon_{2}+\epsilon_{1}\right)}\right\} \\
& \frac{\gamma}{\omega_{\mathrm{v}}}=\frac{1}{2} \alpha_{\mathrm{v}} \operatorname{Im}\left\{\frac{\left(\epsilon_{2}-\epsilon_{1}\right) /\left(\epsilon_{2}+\epsilon_{1}\right)}{4 d^{3} \epsilon_{1}-\alpha_{\mathrm{e}}\left(\epsilon_{2}-\epsilon_{1}\right) /\left(\epsilon_{2}+\epsilon_{1}\right)}\right\}
\end{aligned}
$$

(point model, local metal).

In this case when $\epsilon_{2}$ is very large, the formula for the shift reduces to that given, e.g., in eq. (20) of ref. [4], corresponding to a perfect metal. If in addition we take $\alpha_{\mathrm{e}}$ to be zero, we recover the formula given in eq. (8) of ref. [1].

In calculating the shift and broadening from (22) and (23) we used the SCIB model with the electronic contributions described by the Lindhard dielectric function modified to take into account finite electron lifetime and with the core polarization contribution described by a phenomenological dielectric constant $\epsilon_{b}(\omega)$. For details of how this is done see ref. [9], specifically eqs. (43) and (38) of that reference. In fig. 2 we give numerical results for the shift and broadening versus distance for the case of $\mathrm{CO}$ adsorbed on $\mathrm{Cu}$ with $\epsilon_{1}=1$. For the molecule we have chosen $\alpha_{\mathrm{e}}=2.6 \AA^{3}, \alpha_{\mathrm{v}}=0.16 \AA^{3}, \omega_{\mathrm{v}}=2143 \mathrm{~cm}^{-1}[11,12]$. We rather arbitrarily chose the molecular radius $a=1 \AA$; the essential features of the results are not sensitive to the precise value of the radius. For the metal the parameters were determined as follows. From the optical (infrared) data of 
Johnson and Christy [13] $\epsilon_{\mathrm{b}}=6.5$ and $\omega_{\mathrm{p}}=8.73 \mathrm{eV}$ were determined from a least-squares fit to the form $\epsilon(\omega)=\epsilon_{b}-\omega_{p}^{2} / \omega^{2}$. The electron scattering rate $\nu=0.05 \mathrm{eV}$ was determined from more recent optical data [14]. The Fermi wavevector was determined from the electron density of $\mathrm{Cu}$ using $k_{\mathrm{F}}=$ $\left(3 \pi^{2} n\right)^{1 / 3}=1.36 \AA^{-1}$. The Fermi velocity was determined from the slope of the calculated conduction band [15] at the Fermi surface, $v_{\mathrm{F}}=(\mathrm{d} E / \mathrm{d} k)_{k-k_{\mathrm{F}}}$ $=0.008 \mathrm{c}$. The results using these parameters are shown in fig. 2 in the curves labeled $\epsilon_{\mathrm{b}}=6.5$. To illustrate the importance of the core polarization contribution we also show in fig. 2 curves calculated with the same parameters as above excepting for $\epsilon_{\mathrm{b}}=1$ in one case and $\epsilon_{\mathrm{b}}=2.4$ in the other. The case $\epsilon_{\mathrm{b}}=1$ corresponds to a jellium calculation with no core polarization, while $\epsilon_{b}=2.4$ is an intermediate case determined using the formula $\left(\epsilon_{b}-1\right) /\left(\epsilon_{b}+2\right)=$ $16 \pi\left(a_{\text {core }} / a_{\text {lattice }}\right)^{3}$, where $a_{\text {core }}=0.96 \AA$ is the ionic core radius and $a_{\text {lattice }}=$ $3.61 \AA$ is the fcc lattice constant for $\mathrm{Cu}$ [16]. It is clear from these results that the inclusion of core polarization has an important effect. Of course, the core polarization strictly speaking is itself nonlocal and is screened at very large wavevectors. But this occurs at wavevectors $k \gg a^{-1}$, where $a$ is the ion core radius $(\sim 1 \AA)$. This is beyond the range of wavevectors important in our calculation; the local approximation for $\epsilon_{\mathrm{b}}$ is therefore appropriate. Before we

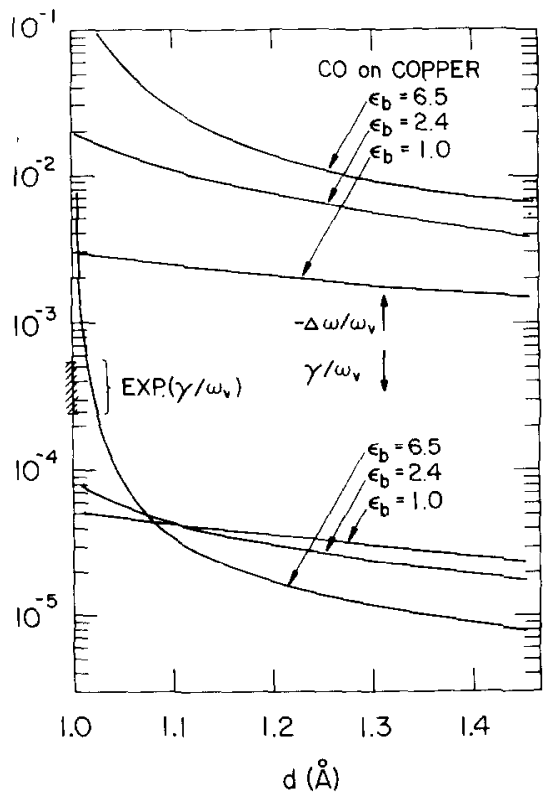

Fig. 2. Calculated results for the electromagnetic shift and broadening for $\mathrm{CO}$ on $\mathrm{Cu}$. Parameters are described in the text. The experimental value for the broadening is indicated by the cross-hatched region on the vertical axis [20]. 
compare these results with experiment we consider in the next section other contributions to the shift and broadening.

\section{Other contributions to the shift}

Here we calculate the mechanical shift in the vibrational frequency of a diatomic model due to its binding to the metal surface. We assume the molecule is oriented normal to the surface. We represent the bonding between the atoms in the free molecule by a spring with force constant $k$ and the bonding of the adsorbed molecule to the metal by a spring with force constant $k_{a}$, as in fig. 3. For the masses we have adopted a notation corresponding to $\mathrm{CO}$ adsorbed on $\mathrm{Cu}$. That is, $m_{\mathrm{O}}$ would be the mass of the oxygen atom, $m_{\mathrm{C}}$ that of the carbon, and $M$ is an indeterminate mass representing the metal. If we think of the adsorbed molecule being bonded to a single $\mathrm{Cu}$ atom, then $M$ is the mass of the $\mathrm{Cu}$ atom; on the other hand, $M$ could be very large if the molecule is bound to a complex of metal atoms. The calculation of the normal modes for this model is straightforward. The normal mode frequencies are roots of the secular equation,

$\omega^{4}-\left(\frac{m_{\mathrm{O}}+m_{\mathrm{C}}}{m_{\mathrm{O}} m_{\mathrm{C}}} k+\frac{m_{\mathrm{C}}+M}{m_{\mathrm{C}} M} k_{\mathrm{a}}\right) \omega^{2}+\frac{m_{\mathrm{O}}+m_{\mathrm{C}}+M}{m_{\mathrm{O}} m_{\mathrm{C}} M} k k_{\mathrm{a}}=0$.

Setting $k_{\mathrm{a}}=0$, we see that the vibrational frequency of the free molecule is

$\omega_{\mathrm{v}}=\left(\frac{m_{\mathrm{O}}+m_{\mathrm{C}}}{m_{\mathrm{O}} m_{\mathrm{C}}} k\right)^{1 / 2}$.

When $k_{\mathrm{a}} \neq 0$, the two roots of (27), which we denote by $\omega_{+}$and $\omega_{-}$, satisfy the relations

$$
\begin{aligned}
& \omega_{+}^{2}+\omega_{-}^{2}=\frac{m_{\mathrm{O}}+m_{\mathrm{C}}}{m_{\mathrm{o}} m_{\mathrm{C}}} k+\frac{m_{\mathrm{C}}+M}{m_{\mathrm{C}} M} k_{\mathrm{a}}, \\
& \omega_{+}^{2} \omega_{-}^{2}=\frac{m_{\mathrm{o}}+m_{\mathrm{C}}+M}{m_{\mathrm{o}} m_{\mathrm{C}} M} k k_{\mathrm{a}} .
\end{aligned}
$$

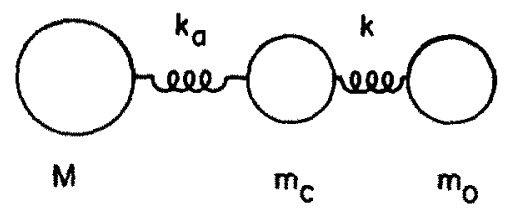

Fig. 3. Coupled mass and spring model used to calculate mechanical shifts. 
Using (28) and eliminating $k_{\mathrm{a}}$ between these last two equations we find $\frac{\omega_{+}^{2}-\omega_{\mathrm{v}}^{2}}{\omega_{\mathrm{v}}^{2}}=\frac{m_{\mathrm{O}} M \omega_{-}^{2}}{m_{\mathrm{C}}\left(m_{\mathrm{O}}+m_{\mathrm{C}}+M\right) \omega_{\mathrm{v}}^{2}-\left(m_{\mathrm{O}}+m_{\mathrm{C}}\right)\left(m_{\mathrm{C}}+M\right) \omega_{-}^{2}}$.

Here we have chosen $\omega_{-}$to be the lower frequency with $\omega_{+}$the higher frequency, near $\omega_{v}$.

In the case of $\mathrm{CO}$ on $\mathrm{Cu}(100)$ the low frequency $\omega_{-}$, the frequency of the $\mathrm{CO}$ bouncing against the metal, has been measured, $\omega_{-}=343 \mathrm{~cm}^{-1}$ [17]. The vibrational frequency of the free molecule is $\omega_{\mathrm{v}}=2143 \mathrm{~cm}^{-1}$. If we choose $m_{\mathrm{O}}=16, m_{\mathrm{C}}=12$, and $M=63$ (or $M=65$ ), corresponding to the molecule being bonded to a single $\mathrm{Cu}$ atom, we find $\omega_{+}=2170 \mathrm{~cm}^{-1}$. If we choose $M=\infty$, corresponding to the atom being bonded to a large metal complex, we find $\omega_{+}=2182 \mathrm{~cm}^{-1}$. We conclude that this mechanical shift is positive with

$27 \mathrm{~cm}^{-1} \leqslant \Delta \omega=\omega_{+}-\omega_{v} \leqslant 39 \mathrm{~cm}^{-1}$.

We next consider the broadening due to its binding to the metal surface. One could imagine the vibrating molecule exciting phonons in the metal as it bounces against the surface. But the frequency $\omega_{+}$of the high-frequency mode is well above the phonon or ripplon frequencies which would be excited. For example, the Debye frequency, which is a measure of the highest phonon frequency, has the value $\omega_{\mathrm{D}}=236 \mathrm{~cm}^{-1}$ for $\mathrm{Cu}$. This is an order of magnitude less than $\omega_{+}$, so one would expect negligible broadening. On the other hand, the low frequency $\omega$. is much closer to $\omega_{D}$, so one might expect a significant broadening of that mode due to phonon or ripplon emission [18].

Finally, one expects a chemical shift due to a weakening of the $\mathrm{C}-\mathrm{O}$ bond stemming from changes in the electron orbitals when the molecule is hound to the metal surface. Some estimate of this can be obtained from data on vibrational spectra of copper carbonyls, for which there are no electromagnetic cffects. From an analysis of the observed vibrational frequencies for different isotopic species of the molecule $\mathrm{CuCO}$, Huber et al. [19] determine force constants for the bonds. Their results correspond to a chemical shift of about $-160 \mathrm{~cm}^{-1}$. As we shall see below, this is much more negative than the chemical shift one could ascribe to $\mathrm{CO}$ adsorbed on a copper substrate. The conclusion is that the nature of the physisorption bond is surprisingly different from that in the copper carbonyl molecule.

\section{Comparison with experiment and other calculations}

We first base our discussion of the experimental results on the presumption that the broadening of the vibrational line for adsorbed $\mathrm{CO}$ is of electromagnetic origin. In fig. 2 the experimental value for the broadening is indicated by the hatched region on the vertical axis [20]. Of the three curves shown only that 
Table 1

Contributions to vibrational frequency shift for $\mathrm{CO}$ adsorbed on copper

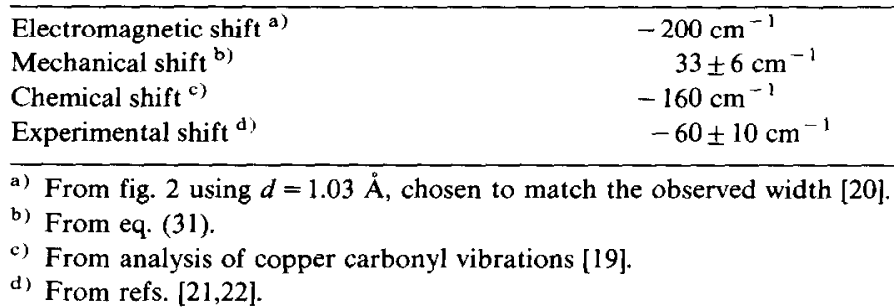

corresponding to $\epsilon_{b}=6.5$ gives sufficient broadening, and then only for $d \leq 1.03 \AA$. This distance corresponds to the sphere nearly touching the surface, and is therefore not unreasonable. We should point out here that the effective position of the metal surface is an adjustable parameter in our calculation, so this value of $d$ should not be taken literally. Choosing therefore $d \equiv 1.03 \AA$ and $\epsilon_{\mathrm{b}}=6.5$ we find the shift is $\Delta \omega / \omega_{\mathrm{v}} \approx-0.1$, or, since $\omega_{\mathrm{v}}=2143$ $\mathrm{cm}^{-1}, \Delta \omega \approx-200 \mathrm{~cm}^{-1}$. In table 1 we give this value for the electromagnetic

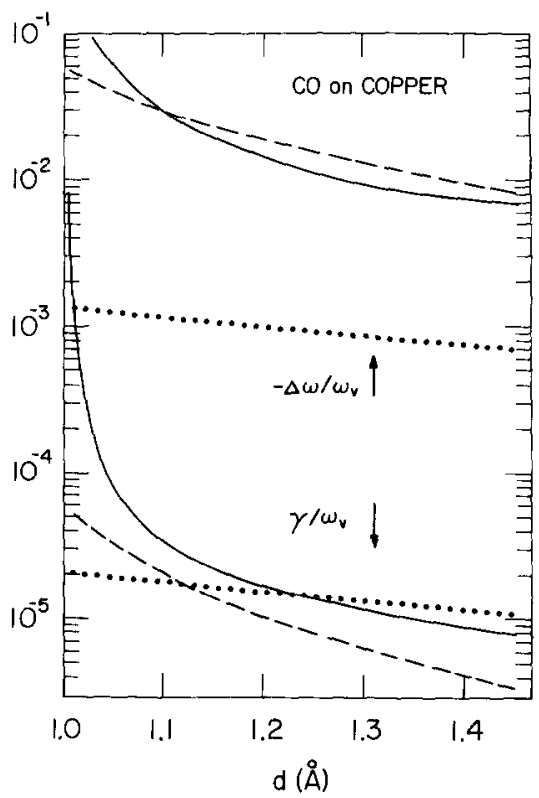

Fig. 4. Comparison of calculations for the shift and broadening from different models. The solid curve is the $\epsilon_{b}=6.5$ curve from fig. 2. The dashed curve is for a local metal with a point-like molecule. The dotted curve is for a nonlocal metal with a point-like molecule but neglecting molecular polarization and core polarization. 
shift together with the expected value of the mechanical and chemical shifts. It is clear that such large electromagnetic and chemical shifts are incompatible with the experimentally observed shift $[21,22]$. We conclude therefore that, although with our models we have shown that it is possible to explain the observed broadening on an electromagnetic basis, it is difficult to explain the observed shift on the same hasis. Since even with no electromagnetic shift the chemical shift is too large and negative, we conclude that the nature of the $\mathrm{C}-\mathrm{O}$ bond in the adsorbed molecule is very different from that in copper carbonyl.

We can reach an alternative conclusion regarding the results in fig. 2 if we choose $d=1.4 \AA$, corresponding to a $\mathrm{C}-\mathrm{O}$ bond length of $1.13 \AA$ [23], a $\mathrm{Cu}-\mathrm{C}$ bond length of $1.82 \AA$ [24], and a metal surface at the edge of the $\mathrm{Cu}$ ion cores (radius $0.96 \AA$ ) [16]. The shift is then $\Delta \omega \approx-17 \mathrm{~cm}^{-1}$ and the half-width $\gamma \approx 0.02 \mathrm{~cm}^{-1}$. Such a narrow linewidth can be understood only if we ascribe the observed width entirely to inhomogeneous broadening.

We compare our results with those of other calculations using the curves shown in fig. 4. The solid curve is a repetition of the curve with $\epsilon_{b}=6.5$ from fig. 2. The dashed curve corresponds to a calculation with a point-like molecule above a local metal, as given by (26), the parameters being otherwise the same as those for fig. 2. Finally the dotted curve is calculated for a point-like molecule above a nonlocal metal but neglecting molecular polarization due to the induced fields, i.e., using (25) with $\alpha_{\mathrm{e}}=0$, and neglecting core polarization, i.e., $\epsilon_{\mathrm{h}}=1$. One should not be misled by the fact that the curve for the local point calculation seems to lie close to that for the much more elaborate calculation with $\epsilon_{\mathrm{b}}=6.5$. In the first place the loss mechanism responsible for the broadening is electron-hole pair production in the nonlocal case and electron scattering in the local case. In fact if in the calculations we were to set $\nu=0$, the numerical results for the width would be little changed in the nonlocal case but would vanish for the local case, and therefore the local description corresponds to an incorrect physical picture. In the second place, the finite size molecule should be placed so that it nearly touches the surface, which means that in the physical situation the curves in the region on the far left of the figure, where the disparities are greatest will be most important.

An important feature of our finite size model which is not present in the other models is the rapid increase in the effects as the molecule approaches the surface. This arises from the strong fields in the gap between the metal and the molecule, resulting from the higher multipole moments induced in the molecule. These higher multipole fields are coupled via the inhomogeneous induced fields to the dipole response, which is thereby strongly enhanced.

Previous calculations are of two kinds. Either a calculation of the shift based on a static approximation which does not allow broadening [1,3-5], or a calculation of the broadening using a model of a point molecule above a nonlocal surface but neglecting molecular polarization (image effects) $[6,7,20]$. 
The first of these would give results for the shift similar to that in the dashed curve in fig. 4 but would give no broadening. The second kind of calculation corresponds to the width in the dotted curve in fig. 4 and would seriously underestimate the shift.

We should make some remarks concerning the use of other models for calculating the reflectivity of the metal surface. One such model is the quantum infinite barrier model, which takes into account the quantum wave functions for the conduction electrons but does not include finite electron lifetime or core polarization. For this model the conduction electron density varies smoothly through the metal surface, in contrast to the abrupt change in the SCIB model. Nevertheless, calculations indicate that this model gives results close to that of our SCIB model with $\epsilon_{\mathrm{b}}=1$ and $\nu=0$ [7]. Calculations by Apell [25] have indicated that, for a dipole far from the surface, a model with a still smoother transition of the conduction electron density through the metal surface will give broadening much greater than that for the SCIB or quantum infinite barrier models. The increased broadening arises from increased coupling to electron-hole excitations in the transition region. But the model he discusses corresponds to jellium with no core polarization. We would argue that core polarization is a feature which necessarily has a sharp profile and that this would reduce the penetration of the fields into the metal. This would significantly reduce the coupling to electron-hole excitations as one sees by comparing the curves in fig. 2 for $d \approx 1.4 \AA$ (where image effects are small).

In conclusion, we wish to point out again what we feel we have learned from our calculation. This is first that finite molecular size and nonlocal metal response are essential ingredients of the calculation of electromagnetic effects. Second that electron-hole excitations give the dominant broadening mechanism of electromagnetic origin, and it is possible that this can explain the observed broadening. Third that core polarization is a significant feature of the metal response. The qualitative nature of these conclusions is in keeping with our view that model calculations such as we have presented are intended to build physical intuition rather than to obtain precise numerical results for comparison with experiment.

\section{References}

[1] F. Delanaye, A.A. Lucas and G.D. Mahan, in: Proc. 7th Intern. Vacuum Congr. and 3rd Intern. Conf. on Solid Surfaces, Vienna, 1977, p. 477.

[2] M. Scheffler, Surface Sci. 81 (1979) 562.

[3] G.D. Mahan and A.A. Lucas, J. Chem. Phys. 68 (1978) 1344.

[4] S. Efrima and H. Metiu, Surface Sci. 92 (1980) 433.

[5] S. Efrima and H. metiu, Surface Sci. 108 (1981) 329.

[6] B.N.J. Persson, J. Phys. C (Solid State Phys.) 11 (1978) 4251.

[7] B.N.J. Persson and M. Persson, Surface Sci. 97 (1980) 609. 
[8] B.N.J. Persson and R. Ryberg, Phys. Rev. Letters 48 (1982) 549.

[9] G.W. Ford and W.H. Weber, Surface. 109 (1981) 451.

[10] W.H. Weber and G.W. Ford, Phys. Rev. Letters 44 (1980) 1774.

[11] P. Hollins and J. Pritchard, Chem. Phys. Letters 75 (1980) 378.

[12] I.L. Fabelinskii, Molecular Scattering of Light (Plenum, New York, 1968) pp. 555-560.

[13] P.B. Johnson and R.W. Christy, Phys. Rev. B6 (1972) 4370.

[14] C.F. Eagen and W.H. Weber, Phys. Rev. B19 (1979) 5068.

[15] H. Ehrenreich and H.R. Philipp, Phys. Rev. 128 (1962) 1622.

[16] N.W. Ashcroft and N.D. Mermin, Solid State Physics (Holt, Reinhart, and Winston, New York, 1976) p. 391.

[17] S. Andersson and B.N.J. Persson, Phys. Rev. Letters 45 (1980) 1421.

[18] R. Hood and H. Metiu, Bull. Am. Phys. Soc. 27 (1982) 227.

[19] H. Huber, E.P. Kündig, M. Moskovits and G.A. Ozin, J. Am. Chem. Soc. 97 (1975) 2097.

[20] B.N.J. Persson and M. Persson, Solid State Commun. 36 (1980) 175.

[21] P. Hollins and J. Pritchard, Surface Sci. 89 (1979) 486.

[22] R.W. Stobie, B. Rao and M.J. Dignam, Surface Sci. 56 (1976) 334.

[23] G. Herzberg, Spectra of Diatomic Molecules (Van Nostrand, New York, 1950) p. 522.

[24] D.P. Graddon, An Introduction to Coordination Chemistry (Pergamon, New York, 1961) p. 71.

[25] P. Apell, Phys. Scripta 24 (1981) 795. 